\title{
On the convergence of double Sumudu transform
}

\author{
Zulfiqar Ahmed $^{\mathrm{a}, *}$, Muhammad Imran Idrees ${ }^{\mathrm{b}}$, Fethi Bin Muhammad Belgacem ${ }^{\mathrm{c}}$, Zahida Perveen ${ }^{\mathrm{b}}$ \\ ${ }^{a}$ Department of Computer Science, GIFT University, Gujranwala, Pakistan. \\ ${ }^{b}$ Department of Mathematics, Lahore Garrison University, DHA, Phase VI, Lahore, Pakistan. \\ ${ }^{c}$ Department of Mathematics, Faculty of Basic Education, PAAET, Shaamyia, Kuwait.
}

\begin{abstract}
In this article, we have studied the convergence properties of double Sumudu transformation, and we presented the results in the form of theorems on convergence, absolute convergence, and uniform convergence of Double Sumudu transformation. The Double Sumudu transform of double Integral has also been discussed for integral evaluation. Finally, we have solved a Volterra integro-partial differential equation by using Double Sumudu transformation.
\end{abstract}

Keywords: Double Sumudu transform, inverse Sumudu transform, integro-partial differential equations.

2010 MSC: 44A05, 34A25.

(C)2020 All rights reserved.

\section{Introduction}

Integral transforms are valuable for the simplification that they bring about, most often in dealing with differential equation subject to particular boundary conditions. The proper choice of class of transformation usually makes it possible to convert not only the derivatives in an intractable differential equation but also the boundary values into terms of an algebraic equation that can be easily solved. The solution obtained is, of course, the transform of the solution of the original differential equation, and it is necessary to invert this transform to complete the operation $[1,7,8,13]$.

Integral transform, is a mathematical operator that produces a new function $f(y)$ by integrating the product of an existing function $F(x)$ and a so-called kernel function $K(x, y)$ between suitable limits. The process, which is called transformation, is symbolized by the equation $f(y)=\int K(x, y) F(x) d x$. Several transforms are commonly named for the mathematicians who introduced them. In the Laplace transform, the kernel is $e^{-x y}$ and the limits of integration are zero and plus infinity, in the Fourier transform, the kernel is $(2 \pi)^{-1 / 2} e^{-i x y}$ and the limits are minus and plus infinity. In [15], The Laplace transform of $f$ is

$$
F(s)=\mathcal{L}(f(t))=\int_{0}^{\infty} e^{-s t} f(t) d t=\lim _{n \rightarrow \infty} \int_{0}^{\tau} e^{-s t} f(t) d t,
$$

\footnotetext{
${ }^{*}$ Corresponding author

Email addresses: dr.zulfiqar.ahmed@hotmail.com (Zulfiqar Ahmed), hefacademy@gmail.com (Muhammad Imran Idrees), fbmbelgacem@gmail.com (Fethi Bin Muhammad Belgacem), drzahida95@gmail.com (Zahida Perveen)
}

doi: $10.22436 /$ jnsa.013.03.04

Received: 2018-12-25 Revised: 2019-09-01 Accepted: 2019-09-04 
whenever the limit exists (as a finite number). When it does, the above integral is said to converge. If the limit does not exist, the integral is said to diverge and there is no Laplace transform defined for $f$. The notation $L(f)$ will also be used to denote the Laplace transform of $f$, and the integral is the ordinary Riemann (improper) integral. The parameter $s$ belongs to some domain on the real line or in the complex plane.

In $[8,17]$ a new integral transform, called the Sumudu transform defined for functions of exponential order. We consider functions in the set $A$, defined by

$$
A=\left\{f(t) \mid \exists M, \tau_{1} \text {, and /or } \tau_{2}>0 \text {, such that }|f(t)|<M e^{\frac{|t|}{\tau_{j}}} \text {, if } t \in(-1)^{j} \times[0, \infty)\right\} \text {. }
$$

For a given function in the set $A$, the constant $M$ must be finite, while $\tau_{1}$ and $\tau_{2}$ need not simultaneously exist, and each may be infinite. Instead of being used as a power to the exponential as in the case of the Laplace transform, the variable $u$ in the Sumudu transform is used to factor the variable $t$ in the argument of the function $f$. Specifically, for $f(t)$ in $A$, the Sumudu transform is defined by

$$
G(u)=s[f(t)]= \begin{cases}\int_{0}^{\infty} f(u t) e^{-t} d t, & 0 \leqslant u<\tau_{2} \\ \int_{0}^{\infty} f(u t) e^{-t} d t, & -\tau_{1} \leqslant u<0\end{cases}
$$

Belgacem presented the fundamental properties, analytical investigation of the Sumudu transform and applications to integral equations. In [3, 7] Belgacem generalizes all existing Sumudu shifting theorems and recurrence results, also presented applications to convolution type integral equations with focus on production problems and inverse Sumudu transform of a singular function that satisfies the Tauberian theorem, where the Dirac delta function fails.

In $[2,4]$, Laplace transform definition is implemented without resorting to Adomian decomposition nor Homotopy perturbation methods. Authors also applied the natural transform to Maxwell's equations and obtained the transient electric and magnetic field solution. In [10], the Sumudu transform is applied to arbitrary powers Dumont bimodular Jacobi elliptic functions for arbitrary powers. Belgacem [5] applied the Sumudu transform to Bessel's functions and equations. In [9], the Sumudu transform integral equation is solved by continuous integration by parts, to obtain its definition for trigonometric functions.

In [6], Belgacem proposed ideas towards the mathematical investigations of the environmental fitness effects on populations dispersal and persistence. In [12], Belgacem gives a sufficient condition to guarantee the solution of the constant coefficient fractional differential equations by Sumudu transform.

Laplace and Sumudu transforms are convenient mathematical tools for solving differential equations. Sumudu transform is defined by the following formula

$$
S[f(t) ; v]=\frac{1}{v} \int_{0}^{\infty} f(t) e^{-\frac{t}{v}} d t, v \in\left(-\tau_{1}, \tau_{2}\right) .
$$

The variable $v$ in this transform is used to factorize the variable $t$ in the argument of the function $f$. The purpose of this study is to show the applicability of Sumudu transform and its efficiency in solving some convergence theorems.

\section{Convergence theorem of double Sumudu integral}

In this section, we prove the convergence theorem of double Sumudu integral.

Lemma 2.1. If the integral

$$
\frac{1}{s} \int_{0}^{\infty} e^{-\frac{t}{s}} \phi(x, t) d t
$$

converges at $\mathrm{s}=\mathrm{s}_{\mathrm{o}}$, then the integral converges for $\mathrm{s}<\mathrm{s}_{\mathrm{o}}$. 
Proof. Consider the set

$$
\alpha(x, t)=\frac{1}{s_{o}} \int_{0}^{t} e^{-\frac{u}{s_{o}}} \phi(x, u) d u,(o<t<\infty) .
$$

Clearly, $\alpha(x, 0)=0$ and $\lim _{t \rightarrow \infty} \alpha(x, t)$ exists because $\frac{1}{s} \int_{0}^{\infty} e^{-\frac{t}{s}} \phi(x, t) d t$ converges at $s=s_{0}$.

By fundamental theorem of Calculus we have $\alpha_{t}(x, t)=\frac{1}{s_{0}} e^{-\frac{t}{s_{0}}} \phi(x, t)$.

If we choose $\epsilon_{1}$ and $R_{1}$ such that $\left(0<\epsilon_{1}<R_{1}\right)$, then

$$
\frac{1}{s} \int_{\epsilon_{1}}^{R_{1}} e^{-\frac{t}{s}} \phi(x, t) d t=\frac{1}{s} \int_{\epsilon_{1}}^{R_{1}} e^{-\frac{t}{s}} s_{o} \alpha_{t}(x, t) e^{\frac{t}{s_{0}}} d t=\frac{s_{o}}{s} \int_{\epsilon_{1}}^{R_{1}} e^{-\left(\frac{s_{0}-s}{s_{o}}\right) t} \alpha_{t}(x, t) d t .
$$

By using integration by parts

$$
\begin{aligned}
& =\frac{s_{\mathrm{O}}}{s}\left\{\left|e^{-\left(\frac{s_{O}-s}{s s_{\mathrm{O}}}\right) t} \alpha(x, t)\right| \epsilon_{\epsilon_{1}}^{R_{1}}-\int_{\epsilon_{1}}^{R_{1}} \alpha(x, t) e^{-\left(\frac{s_{O}-s}{s s_{\mathrm{O}}}\right) t}\left[-\left(\frac{s_{\mathrm{O}}-s}{s s_{\mathrm{O}}}\right)\right] d t\right\} \\
& =\frac{s_{\mathrm{O}}}{s}\left[e^{-\left(\frac{s_{\mathrm{O}}-s}{s s_{\mathrm{O}}}\right) R_{1}} \alpha\left(x, R_{1}\right)-e^{-\left(\frac{s_{O}-s}{s s_{0}}\right) \epsilon_{1}} \alpha\left(x, \epsilon_{1}\right)+\left(\frac{s_{\mathrm{O}}-s}{s s_{\mathrm{O}}}\right) \int_{\epsilon_{1}}^{R_{1}} \alpha(x, t) e^{-\left(\frac{s_{O}-s}{s s_{\mathrm{O}}}\right) t} d t\right] .
\end{aligned}
$$

Now let $\epsilon_{1} \rightarrow 0$. Both terms on the right which depend on $\epsilon_{1}$ approach a limit and

$$
\frac{1}{s} \int_{0}^{R_{1}} e^{-\frac{t}{s}} \phi(x, t) d t=\frac{s_{o}}{s}\left[e^{-\left(\frac{s_{0}-s}{s s_{0}}\right) R_{1}} \alpha\left(x, R_{1}\right)+\left(\frac{s_{o}-s}{s s_{o}}\right) \int_{0}^{R_{1}} \alpha(x, t) e^{-\left(\frac{s_{0}-s}{s s_{0}}\right) t} d t\right] .
$$

Now let $R_{1} \rightarrow \infty$. If $s<s_{0}$, the first term on the right approaches zero and

$$
\frac{1}{s} \int_{0}^{\infty} e^{-\frac{t}{s}} \phi(x, t) d t=\left(\frac{s_{o}-s}{s^{2}}\right) \int_{0}^{\infty} e^{-\left(\frac{s_{0}-s}{s s_{0}}\right) t} \alpha(x, t) d t, \quad \text { for } s<s_{0}
$$

The given theorem is proved if the integral on the right converges.

Now by using the "Limit test" for convergence (see [18]), for this we have

$$
\lim _{t \rightarrow \infty} t^{2} e^{-\left(\frac{s_{0}-s}{s s_{0}}\right) t} \alpha(x, t)=\left[\lim _{t \rightarrow \infty} \frac{t^{2}}{e^{\left(\frac{s_{0}-s}{s s_{0}}\right) t}}\right]\left[\lim _{t \rightarrow \infty} \alpha(x, t)\right]=0 *\left[\lim _{t \rightarrow \infty} \alpha(x, t)\right]=0=\text { finite. }
$$

Therefore, the integral on right hand side of (2.1) converges for $s<s_{0}$.

Hence the given integral $\frac{1}{s} \int_{0}^{\infty} e^{-\frac{t}{s}} \phi(x, t) d t$ converges for $s<s_{0}$.

Lemma 2.2. If integral

$$
h(x, s)=\frac{1}{s} \int_{0}^{\infty} e^{-\frac{t}{s}} \phi(x, t) d t
$$

converges for $\mathrm{s} \leqslant \mathrm{s}_{\mathrm{o}}$ and integral

$$
\frac{1}{p} \int_{0}^{\infty} e^{-\frac{x}{p}} h(x, s) d x
$$

converges at $\mathrm{p}=\mathrm{p}_{\mathrm{o}}$, then the integral (2.2) converges for $\mathrm{p}<\mathrm{p}_{\mathrm{o}}$.

Proof. Let

$$
\beta(x, s)=\frac{1}{p_{o}} \int_{0}^{x} e^{-\frac{u}{p_{o}}} h(u, s) d u, \quad 0<x<\infty .
$$

Therefore $\beta(0, s)=0$ and $\lim _{x \rightarrow \infty} \beta(x, s)$ exists because $\frac{1}{p} \int_{0}^{\infty} e^{-\frac{x}{p}} h(x, s) d t$ converges at $p=p_{0}$. 
By using fundamental theorem of Calculus, equation (2.3) can be written as $\beta_{x}(x, s)=\frac{1}{p_{o}} e^{-\frac{x}{p_{o}}} h(x, s)$. Choose $\epsilon_{2}$ and $R_{2}$ and so that $0<\epsilon_{2}<R_{2}$,

$$
\begin{aligned}
\frac{1}{p} \int_{\epsilon_{2}}^{R_{2}} e^{-\frac{x}{p}} h(x, s) d x & =\frac{p_{o}}{p} \int_{\epsilon_{2}}^{R_{2}} e^{-\left(\frac{p_{o}-p}{p p_{o}}\right) x} \beta_{x}(x, s) d x \\
& =\frac{p_{o}}{p}\left[e^{-\left(\frac{p_{o}-p}{p_{p_{o}}}\right) R_{2}} \beta\left(R_{2}, s\right)-e^{-\left(\frac{p_{o}-p}{p p_{o}}\right) \epsilon_{2}} \beta\left(\epsilon_{2}, s\right)+\left(\frac{p_{o}-p}{p p_{o}}\right) \int_{\epsilon_{2}}^{R_{2}} e^{-\left(\frac{p_{o}-p}{p_{p}}\right) x} \beta(x, s) d x\right] .
\end{aligned}
$$

Now let $\epsilon_{2} \rightarrow 0$.

$$
\frac{1}{p} \int_{0}^{R_{2}} e^{-\frac{x}{p}} h(x, s) d x=\frac{p_{o}}{p}\left[e^{-\left(\frac{p_{o}-p}{p_{p}}\right) R_{2}} \beta\left(R_{2}, s\right)+\left(\frac{p_{o}-p}{p p_{o}}\right) \int_{0}^{R_{2}} e^{-\left(\frac{p_{o}-p}{p_{o}}\right) x} \beta(x, s) d x\right] .
$$

Now let $R_{2} \rightarrow \infty$. If $s<s_{0}$, the first term on the right approaches zero,

$$
\frac{1}{p} \int_{0}^{\infty} e^{-\frac{x}{p}} h(x, s) d x=\left(\frac{p_{o}-p}{p^{2}}\right) \int_{0}^{\infty} e^{-\left(\frac{p_{o}-p}{p_{p}}\right) x} \beta(x, s) d x, \quad \text { for } p<p_{o} .
$$

The given theorem is proved if the integral on the right converges. Now by using the Limit test for convergence (see [18]), we consider

$$
\lim _{x \rightarrow \infty} x^{2} e^{-\left(\frac{p_{o}-p}{p_{0}}\right) x} \beta(x, t)=\left[\lim _{x \rightarrow \infty} \frac{x^{2}}{e^{-\left(\frac{p_{0}-p}{p p_{0}}\right) x}}\right]\left[\lim _{x \rightarrow \infty} \beta(x, t)\right]=0 *\left[\lim _{x \rightarrow \infty} \beta(x, t)\right]=0=\text { finite. }
$$

Therefore, the integral on right hand side of (2.2) converges for $p<p_{0}$.

Hence the given integral $\frac{1}{p} \int_{0}^{\infty} e^{-\frac{x}{p}} h(x, s) d x$ converges for $<p_{o}$.

Theorem 2.3. Let $\phi(x, t)$ be a function of two variables continuous in the positive quadrant of the $x \mathrm{t}-\mathrm{plane}$. If the integral

$$
\frac{1}{p s} \int_{0}^{\infty} \int_{0}^{\infty} e^{-\frac{x}{p}-\frac{t}{s}} \phi(x, t) d x d t
$$

converges at $\mathrm{p}=\mathrm{p}_{\mathrm{o}}, \mathrm{s}=\mathrm{s}_{\mathrm{o}}$, then integral converges for $\mathrm{p}<\mathrm{p}_{\mathrm{o}}, \mathrm{s}<\mathrm{s}_{\mathrm{o}}$.

Proof.

$$
\frac{1}{p s} \int_{0}^{\infty} \int_{0}^{\infty} e^{-\frac{x}{p}-\frac{t}{s}} \phi(x, t) d x d t=\frac{1}{p} \int_{0}^{\infty} e^{-\frac{x}{p}}\left\{\frac{1}{s} \int_{0}^{\infty} e^{-\frac{t}{s}} \phi(x, t) d t\right\} d x=\frac{1}{p} \int_{0}^{\infty} e^{-\frac{x}{p}} h(x, t) d x,
$$

where $h(x, s)=\frac{1}{s} \int_{0}^{\infty} e^{-\frac{t}{s}} \phi(x, t) d t$. By Lemma 2.1, integral $\frac{1}{s} \int_{0}^{\infty} e^{-\frac{t}{s}} \phi(x, t) d t$ converges for $s<s_{0}$. Also by Lemma 2.2, integral $\frac{1}{p} \int_{0}^{\infty} e^{-\frac{x}{p}} h(x, s) d x$ converges for $p<p_{o}$. Therefore, the integral in RHS of (2.5) converges for $p<p_{0}, s<s_{0}$.

Hence the integral $\frac{1}{p s} \int_{0}^{\infty} \int_{0}^{\infty} e^{-\frac{x}{p}-\frac{t}{s}} \phi(x, t) d x d t$ converges for $p<p_{o}, s<s_{0}$. This completes the proof of the Theorem 2.3.

Corollary 2.4. If the integral $\frac{1}{\mathrm{ps}} \int_{0}^{\infty} \int_{0}^{\infty} e^{-\frac{\mathrm{x}}{\mathrm{p}}-\frac{\mathrm{t}}{\mathrm{s}}} \phi(x, \mathrm{t}) \mathrm{d} x \mathrm{dt}$ diverges at $\mathrm{p}=\mathrm{p}_{\mathrm{o}}$ and $\mathrm{s}=\mathrm{s}_{\mathrm{o}}$, then the integral diverges at $\mathrm{p}<\mathrm{p}_{\mathrm{o}}$, $\mathrm{s}<\mathrm{s}_{\mathrm{o}}$.

Corollary 2.5. The region of the convergence of the integral $\frac{1}{\mathrm{ps}} \int_{0}^{\infty} \int_{0}^{\infty} e^{-\frac{\mathrm{x}}{\mathrm{p}}-\frac{\mathrm{t}}{\mathrm{s}}} \phi(\mathrm{x}, \mathrm{t}) \mathrm{d} \mathrm{d} \mathrm{dt}$ is the positive quadrant of the xt-plane.

Now we prove absolute convergence of integral. 
Theorem 2.6. If the integral (2.4) converges absolutely at $\mathrm{p}=\mathrm{p}_{\mathrm{o}}, \mathrm{s}=\mathrm{s}_{\mathrm{o}}$, then integral (2.4) converges absolutely for $p \leqslant p_{0}, s \leqslant s_{0}$.

Proof. We know that $e^{-\frac{x}{p_{0}}-\frac{t}{s_{0}}}|\phi(x, t)| \leqslant e^{-\frac{x}{p}-\frac{t}{s}}$ for $\left(p \leqslant p_{o}<\infty, s \leqslant s_{o}<\infty\right)$. Therefore

$$
\frac{1}{s_{\mathrm{o}} p_{\mathrm{o}}} \int_{0}^{\infty} \int_{0}^{\infty} e^{-\frac{x}{p_{\mathrm{o}}}-\frac{\mathrm{t}}{s_{\mathrm{o}}}}|\phi(x, \mathrm{t})| \mathrm{dtd} x \leqslant \frac{1}{\mathrm{ps}} \int_{0}^{\infty} \int_{0}^{\infty} e^{-\frac{x}{\mathrm{p}}-\frac{\mathrm{t}}{\mathrm{s}}}|\phi(x, \mathrm{t})| \mathrm{d} \mathrm{td} \mathrm{x} .
$$

Form given hypothesis, $\frac{1}{\mathrm{ps}} \int_{0}^{\infty} \int_{0}^{\infty} e^{-\frac{x}{\mathrm{p}}-\frac{\mathrm{t}}{\mathrm{s}}}|\phi(x, \mathrm{t})| \mathrm{d} t \mathrm{~d} x$ converges. Hence, we have

$$
\frac{1}{s_{\mathrm{o}} p_{\mathrm{o}}} \int_{0}^{\infty} \int_{0}^{\infty} e^{-\frac{x}{p_{\mathrm{o}}}-\frac{\mathrm{t}}{s_{\mathrm{o}}}}|\phi(x, \mathrm{t})| \mathrm{d} t \mathrm{~d} x
$$

converges for ( $\left.p \leqslant p_{o} s \leqslant s_{o}\right)$. Therefore the integral (2.4) converges absolutely for $\left(p \leqslant p_{o} s \leqslant s_{o}\right)$.

\section{Uniform convergence}

In this section we prove the uniform convergence of double Sumudu transform.

Lemma 3.1. If $g(x, t)=\frac{1}{s_{0}} \int_{0}^{t} e^{-\frac{v}{s_{0}}} f(x, v) d v$ is bounded on $[0, \infty)$, then the Sumudu transform of $f$ with respect to s converges uniformly on $[s, \infty)$ if $s<s_{0}$.

Proof. If $0 \leqslant r \leqslant r_{1}$, then consider,

$$
\frac{1}{s} \int_{r}^{r_{1}} e^{-\frac{t}{s}} f(x, t) d t=\frac{1}{s} \int_{r}^{r_{1}} e^{-\left(\frac{s_{o}-s}{s s_{o}}\right) t} e^{-\frac{t}{s_{o}}} f(x, t) d t=\frac{s_{o}}{s} \int_{r}^{r_{1}} e^{-\left(\frac{s_{0}-s}{s s_{o}}\right) t} g_{t}(x, t) d t .
$$

Using integration by parts

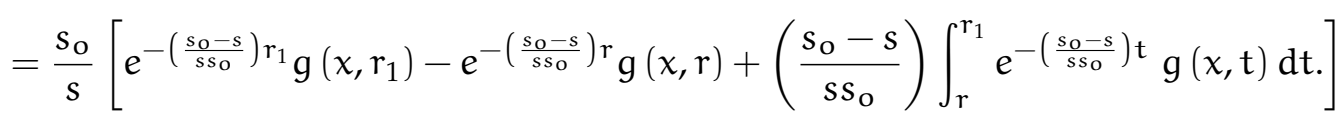

Therefore, if $|g(x, t)| \leqslant M$, then

$$
\begin{aligned}
& \left|\frac{1}{s} \int_{r}^{r_{1}} e^{-\frac{t}{s}} f(x, t) d t\right| \leqslant M\left\{e^{-\left(\frac{s_{O}-s}{s s_{o}}\right) r_{1}}+e^{-\left(\frac{s_{O}-s}{s s_{0}}\right) r}+\left(\frac{s_{o}-s}{s s_{o}}\right) \int_{r}^{r_{1}} e^{-\left(\frac{s_{o}-s}{s s_{0}}\right) t} d t\right\} \\
& =M\left\{e^{-\left(\frac{s_{o}-s}{s s_{0}}\right) r_{1}}+e^{-\left(\frac{s_{0}-s}{s s_{0}}\right) r}-e^{-\left(\frac{s_{0}-s}{s s_{0}}\right) r_{1}}+e^{-\left(\frac{s_{o}-s}{s s_{0}}\right) r}\right\} \\
& =2 \mathrm{Me}^{-\left(\frac{s_{\mathrm{o}}-\mathrm{s}}{s_{\mathrm{o}}}\right) \mathrm{r}} \text { for } \mathrm{s}<\mathrm{s}_{\mathrm{O}} \text {. }
\end{aligned}
$$

By Cauchy criterion for uniform convergence (see [16]), $\frac{1}{s} \int_{r}^{r_{1}} e^{-\frac{t}{s}} f(x, t) d t$ converges uniformly on $[s, \infty)$ if $s<s_{0}$. Hence, Sumudu transform of $f$ with respect to $s$ converges uniformly on $[s, \infty)$ if $s<s_{0}$.

Lemma 3.2. If the integral $g(x, s)=\frac{1}{s} \int_{0}^{\infty} e^{-\frac{t}{s}} f(x, t) d t$ converges uniformly on $[s, \infty)$ if $s<s_{o}$ and $\alpha(x, s)=$ $\frac{1}{p_{\mathrm{o}}} \int_{0}^{\mathrm{x}} \mathrm{e}^{-\frac{\mathrm{u}}{\mathrm{p}_{\mathrm{o}}}} \mathrm{g}(\mathrm{u}, \mathrm{s}) \mathrm{du}$ is bounded on $[0, \infty)$, then the Sumudu transform of $\mathrm{f}$ with respect to s converges uniformly on $[\mathrm{p}, \infty)$ if $\mathrm{p}<\mathrm{p}_{\mathrm{o}}$.

Proof. Proof is similar to Lemma 3.1.

Theorem 3.3. If $\mathrm{f}(\mathrm{x}, \mathrm{t})$ is continuous on $[0, \infty) \times[0, \infty)$ and

$$
H(x, t)=\frac{1}{p_{o} s_{o}} \int_{0}^{x} \int_{0}^{t} e^{-\frac{u}{p_{o}}-\frac{v}{s_{o}}} f(u, v) d u d v
$$

is bounded on $[0, \infty) \times[0, \infty)$, then the double of Sumudu transform of $f$ converges uniformly on $[p, \infty) \times[s, \infty)$ if $\mathrm{p}<\mathrm{p}_{\mathrm{o}}, \mathrm{s}<\mathrm{s}_{\mathrm{o}}$. 
Proof.

$$
\begin{aligned}
H(x, t) & =\frac{1}{p_{o} s_{o}} \int_{0}^{x} \int_{0}^{t} e^{-\frac{u}{p_{o}}-\frac{v}{s_{o}}} f(u, v) d u d v \\
& =\frac{1}{p_{o}} \int_{0}^{x} e^{-\frac{u}{p_{o}}}\left\{\frac{1}{s_{o}} \int_{0}^{t} e^{-\frac{v}{s_{o}}} f(u, v) d v\right\} d u=\frac{1}{p_{o}} \int_{0}^{x} e^{-\frac{u}{p_{o}}} g(u, t) d u,
\end{aligned}
$$

where $g(u, t)=\frac{1}{s_{o}} \int_{0}^{t} e^{-\frac{v}{s_{o}}} f(u, v) d v$ is bounded on $[0, \infty)$.

By Lemma 3.1, Sumudu transform of $f$ with respect to $s$ converges uniformly on $[s, \infty)$ if $s<s_{0}$. Also by Lemma 3.2, Sumudu transform of $g$ with respect to $p$ converges uniformly on $[p, \infty)$ if $p<p_{o}$. Hence double Sumudu transform of $f$ converges uniformly on $[p, \infty) \times[s, \infty)$ if $p<p_{o}, s<s_{o}$.

We now prove the differentiability of double Sumudu transform.

Lemma 3.4. If $g(x, t)=\frac{1}{s_{o}} \int_{0}^{t} e^{-\frac{v}{s_{0}}} f(x, v) d v$ is bounded on $[0, \infty)$, then the Sumudu transform of $f$ is infinitely differentiable with respect to $\mathrm{s}$ on $[\mathrm{s}, \infty)$ if $\mathrm{s}<\mathrm{s}_{\mathrm{o}}$ with

$$
\frac{\partial^{n}}{\partial s^{n}} \bar{f}(x, s)=(-1)^{n} \frac{1}{s} \int_{0}^{\infty} e^{-\frac{t}{s}} t^{n} f(x, t) d t .
$$

Proof. First of all, we will prove that the integrals

$$
I_{n}(x, s)=(-1)^{n} \frac{1}{s} \int_{0}^{\infty} e^{-\frac{t}{s}} t^{n} f(x, t) d t, \quad n=0,1,2,3, \ldots
$$

all converge uniformly on $[s, \infty)$ if $s<s_{o}$ and if $0 \leqslant r \leqslant r_{1}$, then

$$
\begin{aligned}
\frac{1}{s} \int_{r}^{r_{1}} e^{-\frac{t}{s}} t^{n} f(x, t) d t & =\frac{s_{o}}{s} \int_{r}^{r_{1}} e^{-\left(\frac{s_{0}-s}{s s_{0}}\right) t} t^{n} g_{t}(x, t) d t \\
& =\frac{s_{o}}{s}\left[e^{-\left(\frac{s_{0}-s}{s s_{o}}\right) r_{1}} r_{1}^{n} g\left(x, r_{1}\right)-e^{-\left(\frac{s_{0}-s}{s s_{o}}\right) r} r^{n} g(x, r)-\int_{r}^{r_{1}}\left\{\frac{d}{d t} e^{-\left(\frac{s_{o}-s}{s s_{o}}\right) t} t^{n}\right\} g(x, t) d t\right] .
\end{aligned}
$$

Therefore, if $|g(x, t)| \leqslant M<\infty$ on $[0, \infty)$, then

$$
\begin{aligned}
& \left|\frac{1}{s} \int_{r}^{r_{1}} e^{-\frac{t}{s}} t^{n_{1}} f(x, t) d t\right| \leqslant M\left\{e^{-\left(\frac{s_{o}-s}{s s_{0}}\right) r_{1}} r_{1}^{n}+e^{-\left(\frac{s_{o}-s}{s s_{0}}\right) r} r^{n}-e^{-\left(\frac{s_{o}-s}{s s_{o}}\right) r_{1}} r_{1}^{n}+e^{-\left(\frac{s_{o}-s}{s s_{o}}\right) r} r^{n}\right\}, \\
& \left|\frac{1}{s} \int_{r}^{r_{1}} e^{-\frac{t}{s}} t^{n} f(x, t) d t\right| \leqslant 2 M e^{-\left(\frac{s_{o}-s}{s_{o}}\right) r} r^{n} \text { for } 0 \leqslant r \leqslant r_{1} .
\end{aligned}
$$

By Cauchy criterion for uniform convergence, see [16], $I_{n}(x, s)$ converges uniformly on $[s, \infty)$ if $s<$ $s_{\mathrm{o}}$. Now, using [16] and induction proof, we have (3.1). That is Sumudu transform of $f$ is infinitely differentiable with respect to $s$ on $[s, \infty)$ if $s<s_{0}$.

Lemma 3.5. If the integral $\emptyset(x, s)=\frac{1}{s} \int_{0}^{\infty} e^{-\frac{t}{s}} t^{n} f(x, t) d t$ converges uniformly on $[s, \infty)$ if $s<s_{o}$ and

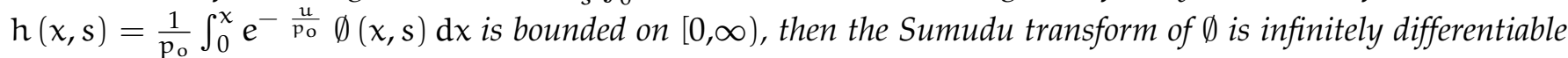
with respect to $p$ on $(p, \infty)$ if $p<p_{o}$, with

$$
\frac{\partial^{\mathrm{m}}}{\partial \mathrm{s}^{\mathrm{m}}} \emptyset(x, \mathrm{~s})=(-1)^{\mathrm{m}} \frac{1}{\mathrm{~s}} \int_{0}^{\infty} e^{-\frac{\mathrm{t}}{\mathrm{s}}} \mathrm{t}^{\mathrm{m}} \emptyset(x, \mathrm{~s}) \mathrm{d} x .
$$

Proof. Proof is similar to Lemma 3.4.

Theorem 3.6. If $\mathrm{f}(\mathrm{x}, \mathrm{t})$ is continuous on $[0, \infty) \times[0, \infty)$ and

$$
\mathrm{H}(\mathrm{x}, \mathrm{t})=\frac{1}{\mathrm{p}_{\mathrm{o}} s_{\mathrm{o}}} \int_{0}^{x} \int_{0}^{\mathrm{t}} e^{-\frac{u}{p_{\mathrm{o}}}-\frac{v}{s_{\mathrm{o}}}} \mathrm{f}(\mathrm{u}, v) \mathrm{dud} v
$$

is bounded on $[0, \infty) \times[0, \infty)$, then the double Sumudu transform of $\mathrm{f}$ is infinitely differentiable with respect to $\mathrm{p}$ 
and $s$ on $[\mathrm{p}, \infty) \times[\mathrm{s}, \infty)$ if $\mathrm{p}<\mathrm{p}_{\mathrm{o}}, \mathrm{s}<\mathrm{s}_{\mathrm{o}}$, with

$$
\frac{\partial^{m+n}}{\partial p^{m} \partial s^{n}} \bar{f}(p, s)=(-1)^{m+n} \frac{1}{p s} \int_{0}^{\infty} \int_{0}^{\infty} e^{-\frac{x}{p}-\frac{t}{s}} x^{m} t^{n} f(x, t) d x d t .
$$

Proof.

$$
\begin{aligned}
H(x, t) & =\frac{1}{p_{o} s_{o}} \int_{0}^{x} \int_{0}^{t} e^{-\frac{u}{p_{o}}-\frac{v}{s_{o}}} f(u, v) d u d v \\
& =\frac{1}{p_{o}} \int_{o}^{x} e^{-\frac{u}{p_{o}}}\left\{\frac{1}{s_{o}} \int_{0}^{t} e^{-\frac{v}{s_{o}}} f(u, v) d v\right\} d u=\frac{1}{p_{o}} \int_{o}^{x} e^{-\frac{u}{p_{o}}} g(u, t) d u,
\end{aligned}
$$

where $g(u, t)=\frac{1}{s_{o}} \int_{0}^{t} e^{-\frac{v}{s_{0}}} f(u, v) d v$ is bounded on $[0, \infty)$.

By Lemma 3.4, Sumudu transform of $f$ is infinitely differentiable with respect to $s$ on $[s, \infty)$ if $s<s_{0}$. Also by Lemma 3.5, Sumudu transform of $g$ is infinitely differentiable with respect to $p$ on $[p, \infty)$ if $p<p_{o}$. Hence double Sumudu transform of $\mathrm{f}$ is infinitely differentiable with respect to $\mathrm{p}$ and $s$ on $[\mathrm{p}, \infty) \times[s, \infty)$ if $s<s_{o}, p<p_{o}$.

\section{Double Sumudu transform of double integral}

We now find the double Sumudu transform of double integral.

Theorem 4.1. If $S_{x} S_{t}\{f(x, t)\}=\bar{f}(p, s)$ and

$$
g(x, t)=\int_{0}^{x} \int_{0}^{t} f(u, v) d v d u
$$

then

$$
S_{x} S_{t}\left\{\int_{0}^{x} \int_{0}^{t} f(u, v) d v d u\right\}=p s \bar{f}(p, s) .
$$

Proof. If we denote $h(x, t)=\int_{0}^{t} f(x, v) d v$, by using fundamental theorem of calculus

$$
h_{t}(x, t)=f(x, t) .
$$

Since

$$
h(x, 0)=0 .
$$

Taking double Sumudu transform of equation (4.2), we get

$$
\bar{h}(p, s)=s \bar{f}(p, s) .
$$

From (4.1), $g(x, t)=\int_{0}^{x} h(u, t) d u$,

$$
g_{x}(x, t)=h(x, t), g(0, t)=0, \quad \bar{g}(p, s)=p \bar{h}(p, s) .
$$

Now by using (4.3) and (4.1), we obtain

$$
S_{x} S_{t}\left\{\int_{0}^{x} \int_{0}^{t} f(u, v) d v d u\right\}=p s \bar{f}(p, s)
$$

\section{Application of double Sumudu transform in Volterra integro-partial differential equation}

We use the double Sumudu transform to solve the problem which is already solved in [14] using differential transform method. 
Example 5.1. Consider the following Volterra integro partial differential equation,

$$
\frac{\delta u(x, y)}{\delta x}+\frac{\delta u(x, y)}{\delta y}=-1+e^{x}+e^{y}+e^{x+y}+\int_{0}^{x} \int_{0}^{y} u(r, t) d r d t,
$$

subject to the initial conditions as:

$$
u(x, 0)=e^{x} \text { and } u(0, y)=e^{y} .
$$

Applying double Sumudu transform of equation (5.1), we get

$$
\frac{1}{p} \bar{u}(p, s)-\frac{1}{p} \bar{u}(0, s)+\frac{1}{s} \bar{u}(p, s)-\frac{1}{s} \bar{u}(p, 0)=-1+\frac{1}{1-p}+\frac{1}{1-s}+\frac{1}{(1-s)(1-p)}+p s \bar{u}(p, s) .
$$

The single Sumudu transforms of equation (5.2) are

$$
\bar{u}(p, 0)=\frac{1}{(1-p)} \text { and } u(0, s)=\frac{1}{(1-s)} .
$$

Substituting (5.4) in (5.3) and simplifying, we obtain

$$
\frac{1}{p} \bar{u}(p, s)-\frac{1}{p(1-s)}+\frac{1}{s} \bar{u}(p, s)-\frac{1}{s(1-p)}=-1+\frac{1}{1-s}+\frac{1}{1-p}+\frac{1}{(1-s)(1-p)}+p s \bar{u}(p, s) .
$$

So $\bar{u}(p, s)=\frac{1}{(1-s)(1-p)}$. Now by using double inverse Sumudu transform, we obtain solution of (5.1) as

$$
u(x, y)=e^{x+y}
$$

\section{Conclusion}

In this paper, we presented convergence, absolute convergence and uniform convergence of double Sumudu transform. Besides these, we obtained double Sumudu transform of double integral and use it to solve Volterra integro-partial differential equation.

\section{References}

[1] Z. Ahmed, M. Kalim, A new transformation technique to find the analytical solution of general second order linear ordinary differential equation, Int. J. Adv. Appl. Sci., 5 (2018), 109-114. 1

[2] F. B. M. Belgacem, Introducing and analysing deeper Sumudu properties, Nonlinear Stud., 13 (2006), 23-41. 1

[3] F. B. M. Belgacem, Applications of Sumudu transform to indefinite periodic parabolic equations, 6th International Conference on Mathematical Problems in Engineering and Aerospace Sciences (Cambridge, U. K.), 2007 (2007), 51-60. 1

[4] F. B. M. Belgacem, Sumudu applications to Maxwell's equations, PIERS Online, 5 (2009), 355-360. 1

[5] F. B. M. Belgacem, Sumudu transform applications to Bessel's Functions and Equations, Appl. Math. Sci., 4 (2010), 3665-3686. 1

[6] F. B. M. Belgacem, E. H. N. Al-Shemas, Towards a Sumudu based estimation of large scale disasters environmental fitness changes adversely affecting population dispersal and persistence, AIP Conference Proceedings, 2014 (2014), 1442-1449. 1

[7] F. B. M. Belgacem, A. A. Karaballi, Sumudu transform fundamental properties investigations and applications, J. Appl. Math. Stoch. Anal., 2006 (2006), 23 pages. 1

[8] F. B. M. Belgacem, A. A. Karaballi, S. L. Kalla, Analytical investigations of the Sumudu transform and applications to integral production equations, Math. Probl. Eng., 2003 (2003), 103-118. 1

[9] F. B. M. Belgacem, R. Silabarasan, A distinctive Sumudu treatment of trigonometric functions, J. Comput. Appl. Math., 312 (2017), 74-81. 1

[10] F. B. M. Belgacem, R. Silambarasan, Sumudu transform of Dumont bimodular Jacobi elliptic functions for arbitrary powers, AIP Conference Proceedings, 2017 (2017), 13 pages. 1

[11] R. R. Dhunde, G. L. Waghmare, On Some Convergence Theorems of Double Laplace Transform, J. Infor. Math. Sci., 6 (2014), 45-54. 
[12] P. Goswami, F. B. M. Belgacem, Solving Special Fractional Differential Equations by Sumudu Transform, AIP Conference Proceedings, 2012 (2012), 111-115. 1

[13] M. I. Idrees, Z. Ahmed, M. Awais, Z. Perveen, On the convergence of double Elzaki transform, Int. J. Adv. Appl. Sci., 5 (2018), 19-24. 1

[14] M. Mohseni Moghadam, H. Saeedi, Application of differential transforms for solving the Volterra integro-partial differential equations, Iran. J. Sci. Technol. Trans. A Sci., 34 (2010), 59-70. 5

[15] J. L. Schiff, The Laplace Transform: Theory and Applications, Springer-Verlag, New York, (1999). 1

[16] W. F. Trench, Functions defined by Improper Integrals, Trinity University (Department of Mathematics), San Antonio, (2012). 3, 3

[17] G. K. Watugala, Sumudu transform - a new integral transform to solve differential equations and control engineering problems, Math. Engrg. Indust., 6 (1998), 319-329. 1

[18] D. V. Widder, Advanced Calculus, Prentice-Hall, Englewood Cliffs, (1961). 2, 2 